\title{
Universal method for magnetic skyrmion bubble generation by controlling the stripe domain instability
}

\author{
Kyoung-Woong Moon (1)', Seungmo Yang', Tae-Seong Ju',2, Changsoo Kim $\mathbb{D}^{1}$, Byoung Sun Chun', \\ Sungkyun Park ${ }^{2}$ and Chanyong Hwang (iD ${ }^{1}$
}

\begin{abstract}
Magnetic skyrmions, which are topological swirling spin textures, have drawn much attention in spintronics because of their use as an information carrier with distinct robustness rooted in their topological nature. Real-time generation of skyrmions is therefore imperative for realizing skyrmion-based spintronic devices. However, to date, experimental demonstration has been limited to exquisite works with well-tuned samples. Here, we report a method to generate skyrmions by driving the stripe instability via an in-plane magnetic field. We have demonstrated that the key parameter determining the stripe domain instability is the stripe width, regardless of other material parameters. This skyrmion generation method can be applicable to generic magnetic films with perpendicular magnetic anisotropy. Our work will facilitate the development of skyrmion-based devices by offering a general method for controlling a large skyrmion population.
\end{abstract}

\section{Introduction}

Magnetic films with a quality factor exceeding 1 show perpendicular magnetic anisotropy (PMA) that favors magnetization in two directions (up or down) perpendicular to the film ${ }^{1}$. Recently, the formation of magnetic topological defects in PMA systems has drawn much interest because topological defects can present a topologically protected magnetization texture known as a magnetic skyrmion ${ }^{2-4}$. Because of their distinct topological properties ${ }^{2-8}$, skyrmions are treated as ideal information carriers for memory ${ }^{9,10}$, logic ${ }^{11,12}$, and neuromorphic devices ${ }^{13}$. Skyrmions have been observed in various magnetic systems ${ }^{14,15}$, even at room temperature $^{16}$. Furthermore, skyrmions have been intentionally created using diverse methods, including a pulsed local

Correspondence: Chanyong Hwang (cyhwang@kriss.re.kr)

'Quantum Spin Team, Korea Research Institute of Standards and Science, Daejeon 34113, Republic of Korea

${ }^{2}$ Department of Physics, Pusan National University, Busan 46241, Republic of Korea

These authors contributed equally: Kyoung-Woong Moon, Seungmo Yang magnetic field ${ }^{14}$ or spin-orbit torque (SOT)-based perturbation $^{17-19}$. However, to date, skyrmions have only been discovered in extremely narrow ranges of material parameters. For example, more than ten multilayers of [heavy metal/ferromagnet/heavy metal] are required to induce a large dipole field ${ }^{20}$, and the ability to control the thickness within $0.1 \mathrm{~nm}$ or less is essential for achieving a particular PMA value ${ }^{5,21}$. Furthermore, these creations of magnetic skyrmions reported thus far are mostly based on the local skyrmion nucleation process occurring at defects with reduced anisotropy, which disturbs their motion after creation and thereby increases the critical threshold for driving skyrmions. These restrictions in creating skyrmions are one of the challenging issues in skyrmion research. In addition, several works have reported the creation of multiple bubble domains after applying a tilted magnetic field, where the specific mechanism remains unclear $^{22,23}$. Furthermore, although several reports have demonstrated a nearly perfect skyrmion lattice in helimagnets $^{24,25}$, a universal way of creating a large number of skyrmions in a typical PMA film system is not yet

\section{(c) The Author(s) 2021}

(c) (i) Open Access This article is licensed under a Creative Commons Attribution 4.0 International License, which permits use, sharing, adaptation, distribution and reproduction cc) in any medium or format, as long as you give appropriate credit to the original author(s) and the source, provide a link to the Creative Commons license, and indicate if changes were made. The images or other third party material in this article are included in the article's Creative Commons license, unless indicated otherwise in a credit line to the material. If material is not included in the article's Creative Commons license and your intended use is not permitted by statutory regulation or exceeds the permitted use, you will need to obtain permission directly from the copyright holder. To view a copy of this license, visit http://creativecommons.org/licenses/by/4.0/. 
available. Therefore, for further in-depth research on magnetic topological physics and realization of a skyrmion application device, more generalized skyrmion generation, as well as a detailed creation mechanism, is needed.

Here, we present a universal method for magnetic skyrmion generation with experimental proof of the microscopic mechanism of skyrmion generation. The current results provide a solution to the unclear mechanism in several works ${ }^{22,23}$ that reported the creation of multiple bubble domains after applying a tilted magnetic field. More importantly, the presented method can be applied to samples with a much broader range of material parameters, such as PMA and the Dzyaloshinskii-Moriya interaction $(\mathrm{DMI})^{26,27}$, compared with previous works. For example, we succeeded in generating many skyrmions even in a high-PMA sample. To date, it is known that such high-PMA samples show large domains ${ }^{27}$ and do not produce individual skyrmions. We believe that by solving the critical problems that have been hampering skyrmion research and applications, our findings will open new pathways for skyrmion-based information processing devices and will help us understand the fundamental physics of skyrmion generation and annihilation.

The stack of our sample $\left(\mathrm{SiO}_{2} / \mathrm{W} / \mathrm{CoFeB} / \mathrm{Ta} / \mathrm{MgO} / \mathrm{Ta}\right)$ with PMA is shown in Fig. 1a, where the PMA originates from the $\mathrm{CoFeB} / \mathrm{MgO}$ interface ${ }^{28,29}$. To manipulate the PMA in this sample, we inserted a Ta wedge layer between $\mathrm{CoFeB}$ and $\mathrm{MgO}$. Unlike the PMA, the total summed magnetization of this system was dominated by the volume of $\mathrm{CoFeB}$, and it barely depended on the insertion of a Ta layer. As a result, it is possible to observe the effect of PMA manipulation by measuring the magnetic domain patterns as a function of the Ta thickness (see Figs. S1, S2 for PMA measurement and Figs. S3, S4 for saturation magnetization measurement). In addition, as the DMI comes from the interface between the W and $\mathrm{CoFeB}$ layers, the insertion of a Ta layer does not significantly change the DMI (see Figs. S5, S6). The upper images of Fig. 1b present typical domain patterns observed by a magneto-optic Kerr effect microscope at several sample positions having different PMA strengths. Light gray indicates upward $(+z)$ magnetization, and dark gray indicates downward $(-z)$ magnetization. These magnetization states consist of an equal number of up and down domains, and both exhibit a certain width $\left(\lambda_{0}\right.$ in Fig. $1 b)$, which can be clearly shown by a fast Fourier transform (see Fig. S7). This type of magnetization state is known as the "stripe domain"30,31.

The stripe domain has been considered a precursor for the creation of magnetic skyrmions. In the simplest example, application of a perpendicular magnetic field $\left(H_{z}\right.$ in Fig. 1a) can stabilize the bubble state compared to the stripe state. As a result, in a moderate $H_{z}$ range, the bubble state becomes the global minimum energy (GME) state. A further increase in $H_{z}$ causes the uniform magnetization state to become the GME state due to the Zeeman energy. Interestingly, all $H_{z}$ ranges for stabilizing the stripe, bubble, and uniform states are normalized by a certain $H_{z}$ value at which the bubbles disappear $\left(H_{z, \mathrm{~B}}\right)^{30,31}$. Roughly, the stripe domain, bubble and uniform states are at the GME in the regions from 0 to $0.5 H_{\mathrm{z}, \mathrm{B}}, 0.5 H_{\mathrm{z}, \mathrm{B}}$ to $H_{\mathrm{z} \text {, }}$ в and $H_{\mathrm{z}, \mathrm{B}}$ to infinity, respectively. Thus, most studies on skyrmions try to use stripe domain states to create skyrmions. However, to date, these attempts at changing the stripe domains into the bubble state have been limited to narrow stripes.

We will show why creating bubble domains fails for wide stripes under $H_{z}$. We measured the minimum field strength $\left(H_{\mathrm{z}, \mathrm{S}}\right)$ to erase all the stripe domains. The values of $H_{\mathrm{z}, \mathrm{S}}$ were obtained at several sample positions with different values of $\lambda_{0}$. We performed the same experiments with initial bubble domain states, as shown in Fig. 1b, to obtain $H_{\mathrm{z}, \mathrm{B}}$. These $H_{\mathrm{z}, \mathrm{S}}$ and $H_{\mathrm{z}, \mathrm{B}}$ values are plotted in Fig. 1c (blue and red circles). From the measured $H_{\mathrm{z}, \mathrm{B}}$ values, the regions where the stripe and bubble states were the GME states (the orange and green areas in Fig. $1 c$, respectively) could be estimated ${ }^{30-32}$. It is worth noting that the measurement of $H_{\mathrm{z}, \mathrm{B}}$ indicated by red filled circles has never been achieved for wide-stripe domains. Remarkably, the overall results of Fig. 1c are separated at $\lambda_{0}=1.7 \mu \mathrm{m}$. For $\lambda_{0}<1.7 \mu \mathrm{m}$ (narrow stripes), increasing $H_{z}$ cuts all stripe domains into bubble domains at $H_{z, S}$, and the bubble domains are maintained before reaching the uniform state. Figure 1d shows the sequential change in the magnetization state with $H_{z}$ at a narrowstripe position $\left(\lambda_{0}=1.3 \mu \mathrm{m}\right)$. Increasing $H_{z}$ leads to a state change of stripe-bubble-uniform. Blue and red circles between images indicate stripe-bubble and bubbleuniform transitions, respectively. Decreasing $H_{z}$ also induces uniform-bubble-stripe transitions, by nucleation of bubble domains and their elongation. However, in wide stripes $\left(\lambda_{0}>1.7 \mu \mathrm{m}\right)$, we cannot see such a stripe-bubble-uniform transition because all bubble domains already disappeared at a much smaller field than $H_{z, \mathrm{~S}}$. Therefore, we cannot form a bubble state by changing $H_{z}$ in wide stripes. Figure 1e presents this failure for a wide stripe $\left(\lambda_{0}=2.6 \mu \mathrm{m}\right)$; only a strip-uniform transition occurred at large $H_{z}$ values (indicated by a blue circle). When $H_{z}$ was reduced, the bubble domains did not nucleate within the range of the bubble GME state. Instead, the stripe domain grew to fill the entire area under the reduced magnetic field, which is the stripe domain GME state. This event is indicated by a green square in Fig. 1e, c.

To address the question of why bubbles are not easily formed in a wide-stripe domain state, we examined the 


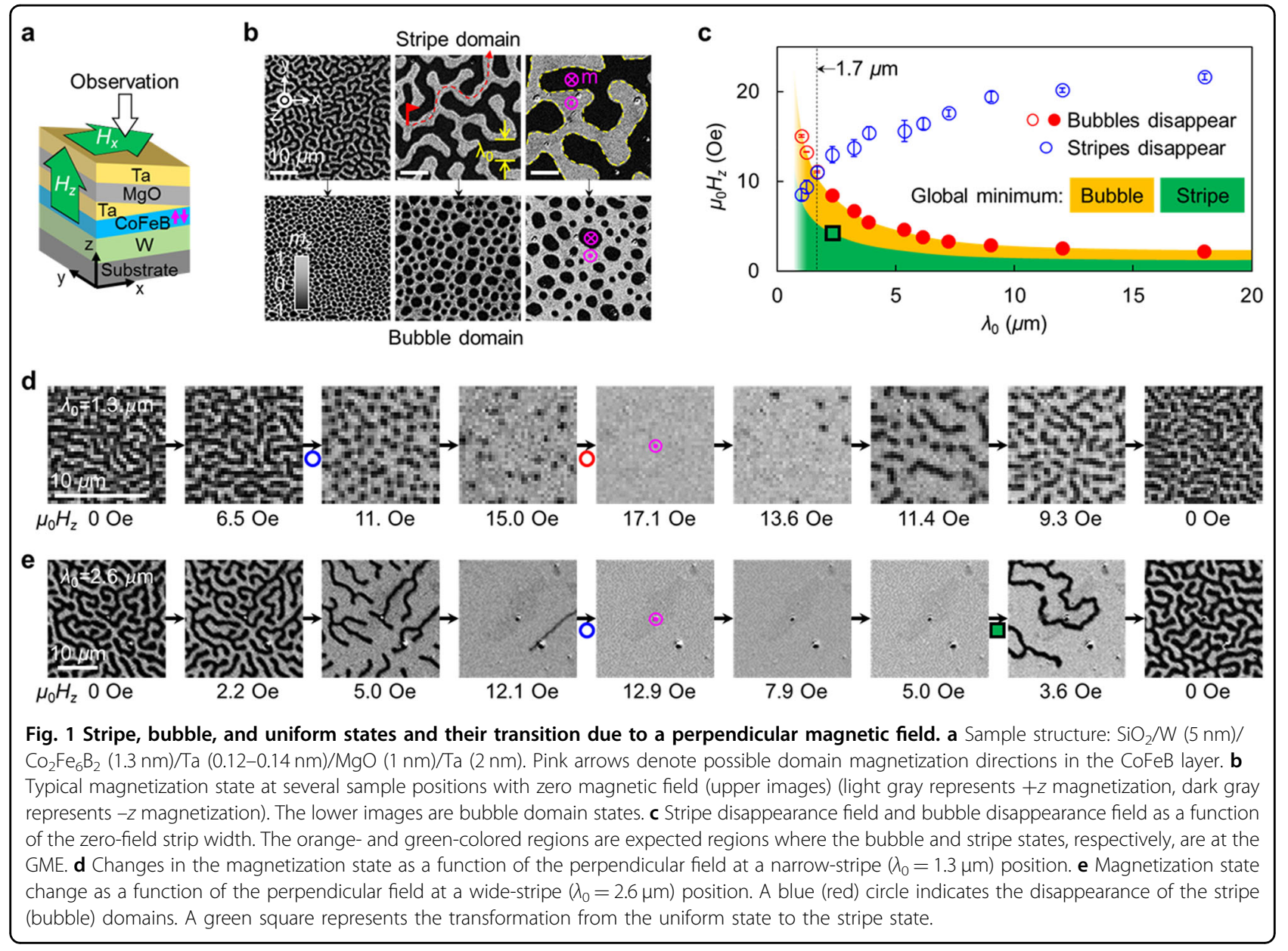

effect of an in-plane magnetic field $\left(H_{x}\right)$. We prepared an initial stripe domain state at zero field. We turned on $H_{x}$ and captured the domain image; then, we turned off $H_{x}$ and captured the image again. Repeating these procedures with increasing $H_{x}$ resulted in the sequential images shown in Fig. 2a. A reduced stripe width was observed with the application of $H_{x}$. However, the stripe width was recovered to the initial width after turning off $H_{x}$. The field-off states showed no isolated domain up to $0.34 \mathrm{kOe}$, and the domain states looked similar to the initial state. Therefore, we can expect that $H_{x}$ induces continuous deformation of the stripe structure by adding narrower stripes (red lines in Fig. 2a) to the original stripe structure (white lines in Fig. 2a).

Interestingly, a discontinuous deformation occurred after applying $H_{x}$ above $0.45 \mathrm{kOe}$. The initial stripe structures almost disappeared, and isolated domains were created (red boxes in Fig. 2a). Further application of $H_{x}$ produced stripe domains almost parallel to the $x$-direction but still left isolated domains. Therefore, the formation of isolated domains can be considered to be strongly related to the stripe width. Thus, we plotted the stripe width $(\lambda)$ as a function of $H_{x}$, as shown in Fig. 2b, at several sample positions with different stripe widths $\left(\lambda_{0}\right)$. The value of $\lambda$ is defined along the $y$-axis (inset of Fig. 2b). The logarithm of the stripe width shows a linear dependence on $H_{x}$ (see Supplementary Note 5).

Our finding is a universal value of the stripe width that creates isolated domains regardless of the initial stripe width. The creation of isolated domains was confirmed by zero-field domain images (see Figs. S8, S9 for the detailed procedure). The results of the required $H_{x}$ and $\lambda$ at each position are plotted together in Fig. $2 \mathrm{~b}$ (black open circles). All the width values are in the range of $1.5-1.8 \mu \mathrm{m}$, and this range is almost the same as the reference value $(1.7 \mu \mathrm{m})$ for dividing the narrow stripes and the wide stripes in Fig. 1c. We expect that sufficiently narrowstripe domains can be cut into small segments and create isolated domains, as shown in Fig. 1d. Thus, it can be seen that the width of the stripe is an important variable in determining the instability of the stripe domain.

The application of an additional field $H_{z}$ during the stripe-cutting process can leave only bubble domain states. It is clear that $H_{x}$ does not distinguish between $+z$ 


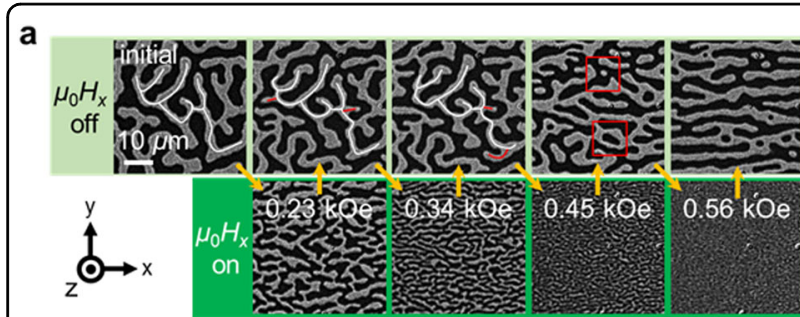

b

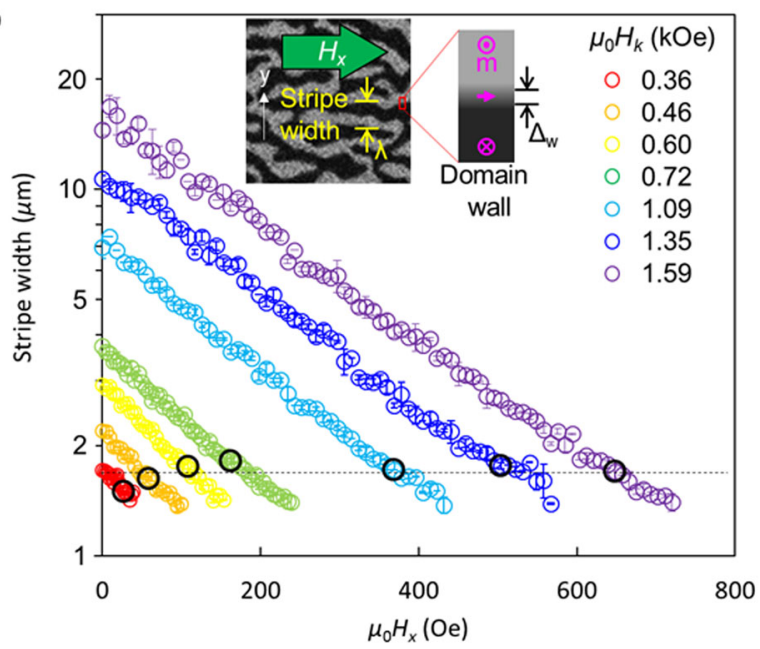

Fig. 2 Effect of an in-plane magnetic field on the stripe domain state. a Sequential images of the magnetization state with varying inplane magnetic field. $\mathbf{b}$ Variation in the stripe domain width as a

function of the in-plane field at several sample positions. $H_{k}$ indicates the strength of the PMA converted to the magnetic field value (see Supplementary Note 1 for measurements of $H_{k}$ ). Black dots indicate the critical values for isolated domain generation at each position (see Supplementary Note 6). The inset shows the definition of the stripe width, the in-plane field direction, and a schematic image of the domain wall structure. The dotted line represents $\lambda=1.7 \mu \mathrm{m}$.

and $-z$ domains, so the numbers of $+z$ and $-z$ isolated domains after application of $H_{x}$ are almost equal (Fig. 2a). In addition, the isolated domains made in this way have irregular shapes and sizes with low density. However, the additional $H_{z}$ can select only one of the $+z$ and $-z$ domains due to the Zeeman energy. As a result, it is expected that as many bubbles of one polarity are formed as possible, and a dense circular bubble state is created for close packing. Figure 3a shows typical domain patterns at zero field after the simultaneous application of $H_{x}$ and $H_{z}$ to the values presented at the center of the squares for $1 \mathrm{~s}$. To obtain these results, we prepared initial wellconnected stripe states using $H_{z}$ for every single image. When $H_{x}$ was $<0.54 \mathrm{kOe}$, a larger $H_{z}$ created uniform magnetization states, and $H_{z}=0$ induced stripe states. This result is similar to those for the wide stripe shown in Fig. 1e. When $H_{x}$ exceeded $0.54 \mathrm{kOe}$, the creation of isolated domains was observed, as were bubble-filled states. The isolated domain number (bubble number) at a
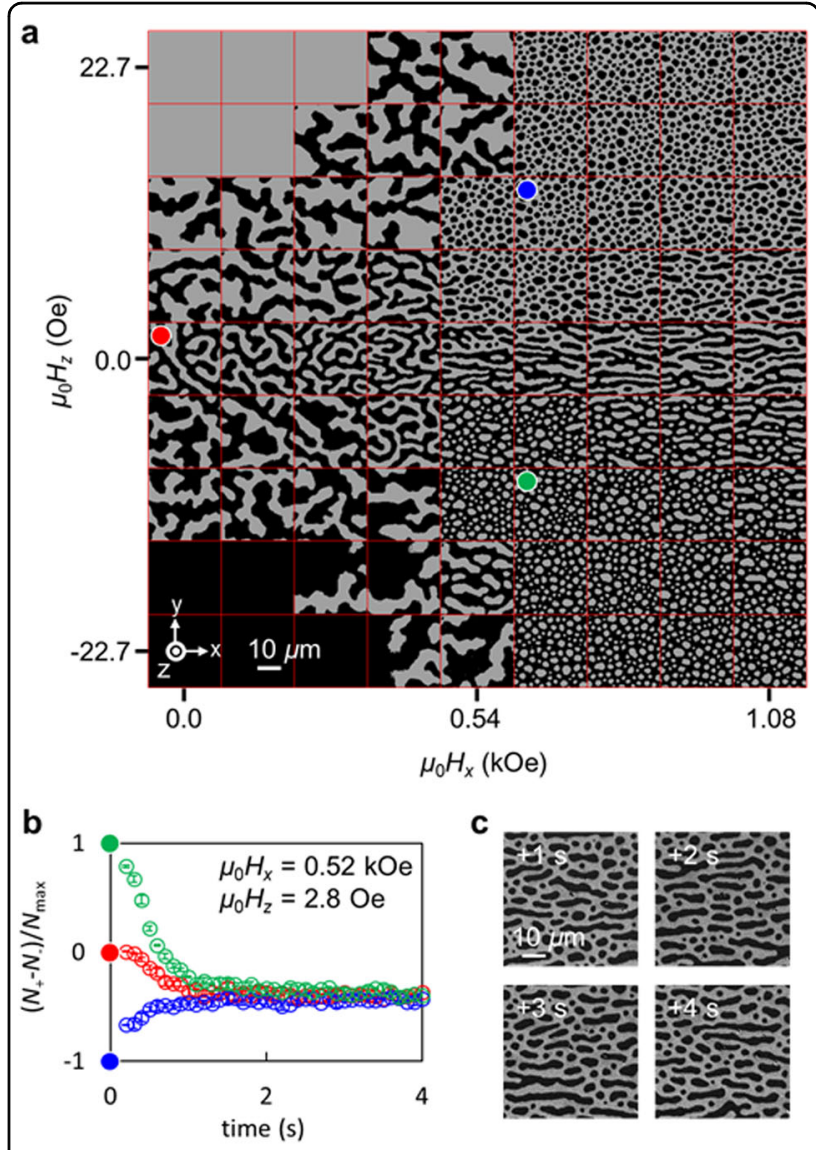

Fig. 3 Magnetization state pattern changes under the combination of an in-plane field and a perpendicular field. a Zero-field magnetization states after application of the magnetic field. The values of $H_{x}$ and $H_{z}$ at the center of each image are the applied field values. $\mathbf{b}$ Convergence of normalized isolated domain numbers as a function of field application time for different initial states. Filled dots at time 0 represent different initial states marked in a. Red, blue, and green filled dots indicate the well-connected stripe state, $-z$ bubble-filled state, and $+z$ bubble-filled state, respectively. $\mathbf{c}$ Magnetization state changes when the magnetic field is applied for a longer time after the number of isolated domains converges.

larger $H_{z}$ is much larger than that of the $H_{z}=0$ state. Therefore, the application of simultaneous $H_{x}$ and $H_{z}$ can cause the bubble-filled states to be the GME states. As a result, the bubble state can be created by simultaneously applying $H_{x}$ and $H_{z}$ in a wide-stripe domain sample.

The detailed process of isolated domain creation will be discussed next. We prepared three different initial domain states, namely, a well-connected stripe state, $\mathrm{a}+z$ bubblefilled state, and a $-z$ bubble-filled state (the colored dots in Fig. 3a). A magnetic field $\left(\mu_{0} H_{z}=2.8 \mathrm{Oe}, \mu_{0} H_{x}=\right.$ $0.52 \mathrm{kOe}$ ) was applied for a certain time, and after that, the isolated domains under zero field were counted. The number of $\pm z$ isolated domains was $N_{ \pm}$, and $N_{\max }$ was the total number of isolated domains of the initial bubblefilled states. The ratio $\left(N_{-}-N_{+}\right) / N_{\max }$ is plotted in Fig. 3b 


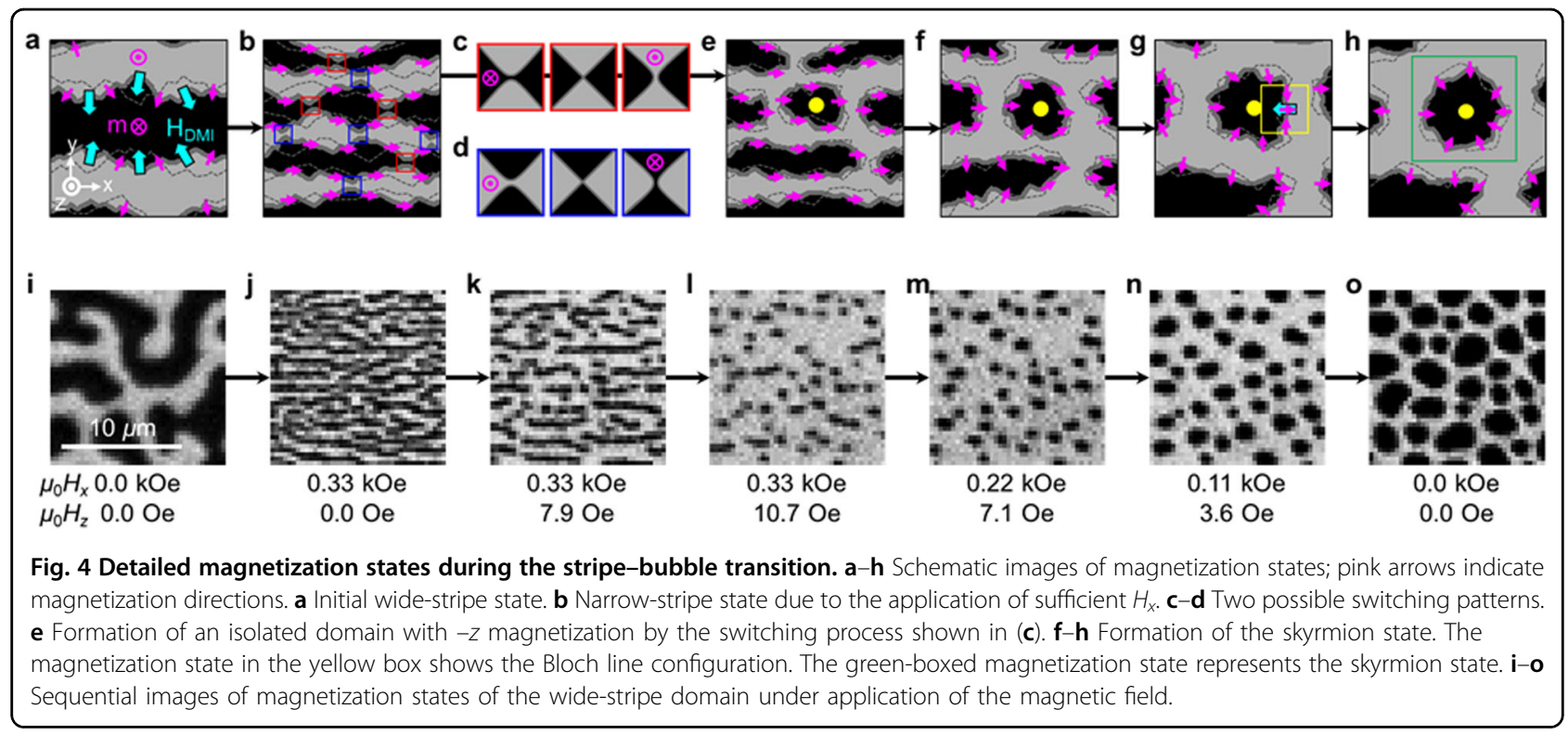

as a function of the field application time. All data exponentially converged to a single value after $2 \mathrm{~s}$. Further application of the field did not change $N_{ \pm}$, although the detailed domain images were not the same. The domain states with additional application of the field are shown in Fig. 3c. Thus, we believe that the converged state is a thermal equilibrium state.

Because of the thermal energy, domain walls randomly fluctuate from an equilibrium position ${ }^{33}$. A schematic situation for this state is shown in Fig. 4a. The domain wall is perturbed by thermal energy and could have a different shape (gray dashed lines) over time. If two adjacent walls are far apart (wide stripes), it is difficult for domain walls to collide with each other (Fig. 4a) ${ }^{31}$. Note that the domain wall magnetization can also have a certain chiral structure due to the DMI. The DMI generates an effective magnetic field ( $H_{\mathrm{DMI}}$, cyan arrows in Fig. 4a) in the domain wall. In our system, the interface between $\mathrm{W}$ and $\mathrm{CoFeB}$ is a meaningful source of the $\mathrm{DMI}^{34,35}$, and it adopts the $(+z)-(+y)-(-z)-(-y)-(+z)$ chiral structure in the $y$-direction and the $(+z)-(+x)-(-z)-(-x)-(+z)$ chiral structure in the $\mathrm{x}$-direction. This chiral magnetization texture can also enhance the stripe stability.

Applying $H_{x}$ reduces the stripe width, and two adjacent domain walls will have more chances of colliding with each other. When these domain walls collide, the form of the domain may change. As shown in Fig. 4b, the domain wall collisions can take two forms (Fig. 4b, red and blue boxed areas). Figure 4c, d depicts these two possible domain pattern change processes. In Fig. $4 \mathrm{c}$, the $-z$ domain connected in the $x$-direction becomes the $+z$ domain connected in the $y$-direction. By contrast, Fig. $4 \mathrm{~d}$ shows the process in which the $+z$ domain connected in the $x$-direction is transformed into the $-z$ domain connected in the $y$-direction. If there is no $H_{z}$, then these two pattern change processes occur equally. However, a nonzero $H_{z}$ breaks the symmetry in the $+z$ and $-z$ domains; thus, $H_{z}$ selects only one of these two collision processes. Thus, the magnetization state in Fig. $4 \mathrm{~b}$ is converted to that in Fig. 4e under $+H_{z}$. This process creates an isolated domain with $-z$ magnetization (the yellow dot in Fig. 4e). If $H_{x} \gg H_{\text {DMI }}$, then all domain wall magnetizations are aligned along $H_{x}$, and thus, the chiralinduced stability disappears. The isolated domain in Fig. $4 \mathrm{e}$ is not a skyrmion because a skyrmion consists of vectors in all directions ${ }^{2}$, but there is no $-x$ magnetization vector in this figure. If $H_{x}$ is reduced, then the magnetization direction of the magnetic domain wall is aligned along the $H_{\mathrm{DMI}}$ direction (Fig. $4 \mathrm{e}-\mathrm{g}$ ). This continual deformation of the magnetization state inevitably forms a Bloch line in the domain wall (yellow box in Fig. $4 \mathrm{~g}$ ) ${ }^{36}$. Such a Bloch line contains an opposite magnetization to $H_{\text {DMI }}$. When $H_{\text {DMI }}$ (cyan arrow in Fig. $4 \mathrm{~g}$ ) is high enough to switch this opposite magnetization or another effect, such as thermal fluctuation, reverses this magnetization, the bubble domain becomes a skyrmion (Fig. $4 \mathrm{~h}$ ).

Figure $4 \mathrm{i}-\mathrm{o}$ depicts sequential magnetization state images showing the conversion process from the initial wide stripes to the bubble-filled state as a function of field application. Figure $4 \mathrm{i}$ shows the initial wide stripes with $\lambda_{0}=4.9 \mu \mathrm{m}$ that are too wide to observe the stripe cutting process. Applying sufficient $H_{x}$ reduces $\lambda$ to $1.2 \mu \mathrm{m}$, and then, the stripe is ready to be broken up (Fig. 4j). The additional $H_{z}$ allows breakage of the $-\mathrm{z}$ stripe domain into -z bubble domains (Fig. 4k, l). Then, by turning off $H_{x}$ and $H_{z}$ at the same time, the bubble-filled state remains. Reducing $H_{x}$ to zero increases the stripe width as well as the bubble size, and reducing $H_{z}$ also assists the expansion 

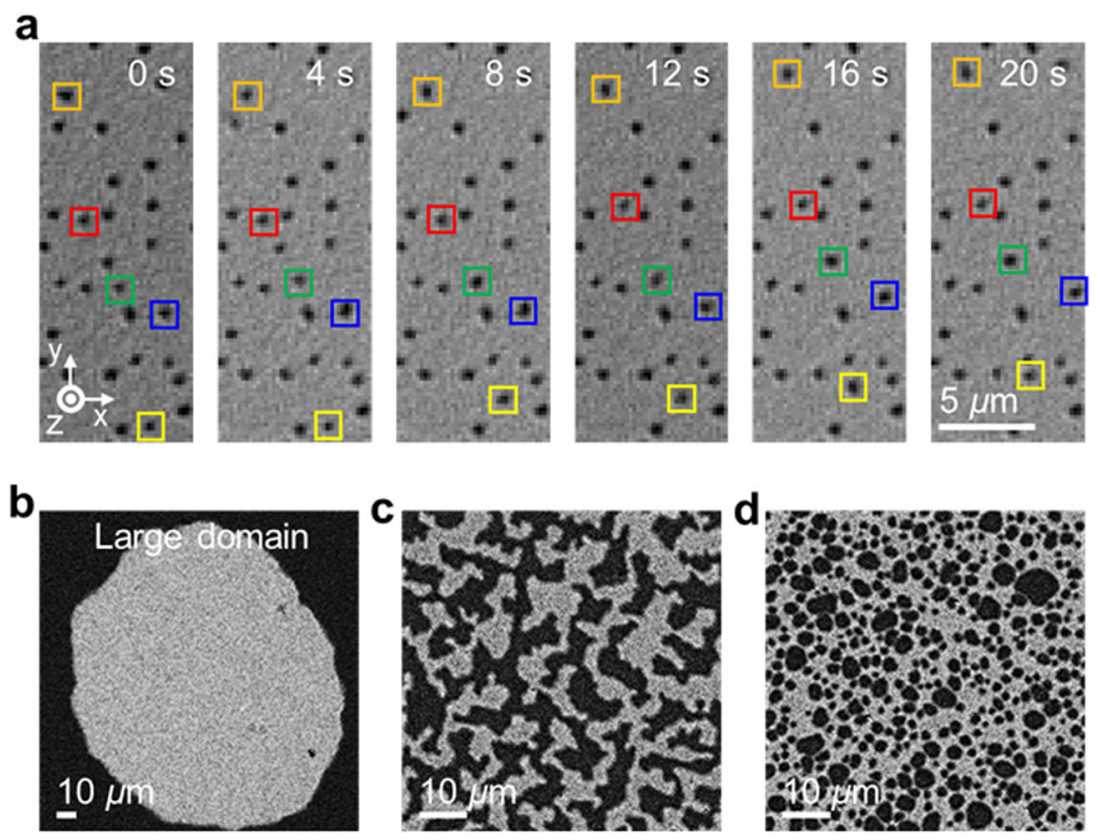

Fig. 5 Application of the creation method of bubble domains. a Current-induced motion of magnetization states. Constant current flow in the $y$ direction. The current density is $5.0 \times 10^{8} \mathrm{~A} \mathrm{~m}^{-2}$, and $\mu_{0} \mathrm{H}_{z}=7 \mathrm{Oe}$. b-d Magnetization states of $\mathrm{SiO}_{2} / \mathrm{Ta}(3 \mathrm{~nm}) / \mathrm{Co}_{4} \mathrm{Fe}_{4} \mathrm{~B}_{2}(1.1 \mathrm{~nm}) / \mathrm{MgO}(1.5 \mathrm{~nm}) / \mathrm{Ta}$ $(2 \mathrm{~nm})$. b Large circular domain formation. c Stripe domain state under $\mu_{0} H_{x}=2.1 \mathrm{kOe}$. d Zero-field magnetization state after application of $\mu_{0} H_{x}=$ $3 \mathrm{kOe}$ and $\mu_{0} H_{z}=10$ Oe.

of bubble domains ${ }^{30,31}$ to finally produce the bubble-filled state, as shown in Fig. 4m-o (see Fig. S10 for constant $H_{\mathrm{x}}$ ). This is an impossible result with narrow-stripe domains because a thermal fluctuation easily finds the GME state. However, in the wide-stripe domain state, the thermal fluctuations have difficulty changing the magnetization state, so the bubble-filled state exists as a local minimum energy state even in the absence of magnetic fields.

Whether the formed bubble is a skyrmion cannot be confirmed by the domain image but is indirectly confirmed by checking the current-induced motion because when the bubble has a skyrmion texture, it is moved by a current $^{37-39}$. The images in Fig. 5a are sequential images of bubble motion after turning a current on in the $y$ direction with $\mu_{0} H_{z}=7$ Oe (also see Supplementary Video 1). $H_{z}$ removed many bubble domains and left a few with quasi-uniform size because compressed domains can easily reach the local minimum energy state (circular bubble domain) due to their small size. Bubble domains that move in the $y$-direction are identified by colored squares. From these results, we think that the spin textures shown are likely to be skyrmions. In addition, it is worth noting that the current for bubble motion is extremely low $\left(5 \times 10^{8} \mathrm{Am}^{-2}\right)$, which can indicate that the observed skyrmions are not domains nucleated from point defects and are therefore freely mobile.

Ours is a universal method for skyrmion creation because bubble-filled states can be created as a seed for skyrmions in arbitrary PMA samples. When the PMA strength is sufficiently large, the stripe width exceeds the finite sample size. Such samples are no longer called stripe domains but large domain samples ${ }^{27}$. It is important that the presented method is applicable to large domains because the domain wall energy reduction by $H_{x}$ is a universal phenomenon in PMA samples. A sample showing a large domain is presented in Fig. 5b. The application of $H_{z}$ switches the magnetization at reduced PMA defects first, and then, the reversed domain expands in all directions, forming a large circular domain (Fig. 5b). However, the application of $H_{x}$ can create observable stripe domains. Figure $5 \mathrm{c}$ shows a magnetization state with $\mu_{0} H_{x}=2.1 \mathrm{kOe}$. Figure $5 \mathrm{~d}$ shows an example of a zero-field state after application of $\mu_{0} H_{x}=3 \mathrm{kOe}$ and $\mu_{0} H_{z}=10$ Oe. It is clear that a bubble-filled state can be created even in a large domain sample.

We have shown that in a prototypical PMA material, $\mathrm{CoFeB}$, a large number of magnetic skyrmions can be easily created by applying a magnetic field tilted from the in-plane direction at room temperature. Our proposed method for the creation of multiple skyrmions can be applied to generic PMA materials and thus overcomes the limitations of previous methods with a narrow range of material parameters. However, we have demonstrated that skyrmions were created using an external magnetic field, where the key parameter determining the instability of the stripe domain is not the field but the width of the 
stripe. From the point of view of applications, the important parameter, the skyrmion density, is also determined by the stripe width (see Fig. S11). Therefore, through control of the width of the stripes by electrical means (e.g., PMA and DMI control using an electric field ${ }^{40,41}$ ), we can create skyrmions without a magnetic field in the future. Our findings will not only facilitate the development of skyrmion-based spintronic devices by offering a universal generation method for multiple magnetic skyrmions but also trigger fundamental research on the hitherto underestimated role of domain instability in the skyrmion phase and its neighboring phases.

\section{Acknowledgements}

This work was supported by the National Research Foundation of Korea (Grant No. 2015M3D1A1070467, 2019M3F3A1A02072478) and a National Research Council of Science and Technology grant (Grant No. CAP-16-01-KIST) from the Korean government (MSIP).

\section{Author contributions}

K.W.M., S.Y., and C.H. conceived the idea. C.H. supervised the execution of the whole work. K.W.M. and S.Y. explained the experimental results. S.Y., K.W.M. and C.K. performed the experiments and image analysis. S.Y. and K.W.M. designed the samples. S.Y., T.S.J., B.S.J. and S.P. fabricated the samples. K.W.M., S.Y. and C. $\mathrm{H}$. wrote the paper. All authors were involved in the discussion of the results and commented on the paper.

\section{Data availability}

All relevant data are available from the authors upon reasonable request.

\section{Conflict of interest}

The authors declare no competing interests.

\section{Publisher's note}

Springer Nature remains neutral with regard to jurisdictional claims in published maps and institutional affiliations.

Supplementary information The online version contains supplementary material available at https://doi.org/10.1038/s41427-021-00290-3.

Received: 15 October 2020 Revised: 7 January 2021 Accepted: 19 January 2021.

Published online: 12 March 2021

\section{References}

1. Malozemoff, A. P. \& Slonczewski, J. C. Magnetic Domain Walls in Bubble Materials. (Academic Press, 1979).

2. Nagaosa, N. \& Tokura, Y. Topological properties and dynamics of magnetic skyrmions. Nat. Nanotechnol. 8, 899-911 (2013).

3. Christian, H. B. et al. The 2020 Skyrmionics Roadmap. J. phys. D. Appl. phys. $\mathbf{5 3}$ 363001 (2020).

4. Bogdanov, A. N. \& Yablonskii, D. A. Thermodynamically stable "vortices" in magnetically ordered crystals. The mixed state of magnets. Zh. Eksp. Teor. Fiz. 95, 178-182 (1989).

5. Jiang, W. et al. Direct observation of the skyrmion Hall effect. Nat. Phys. 13, 162-169 (2017)

6. Litzius, K. et al. Skyrmion Hall effect revealed by direct time-resolved X-ray microscopy. Nat. Phys. 13, 170-175 (2017).

7. Hirata, Y. et al. Vanishing skyrmion Hall effect at the angular momentum compensation temperature of a ferrimagnet. Nat. Nanotechnol. 14, 232-236 (2019).

8. Fert, A., Reyren, N. \& Cros, V. Magnetic skyrmions: advances in physics and potential applications. Nat. Rev. Mater. 2, 17031 (2017).
9. Tomasello, R. et al. Strategy for the design of skyrmion racetrack memories. Sci. Rep. 4, 6784 (2014).

10. Fert, A., Cros, V. \& Sampaio, J. Skyrmions on the track. Nat. Nanotechnol. 8, 152-156 (2013).

11. Luo, S. et al. Reconfigurable Skyrmion Logic Gates. Nano Lett. 18, 1180-1184 (2018).

12. Zhang, X., Ezawa, M. \& Zhou, Y. Magnetic skyrmion logic gates: conversion, duplication and merging of skyrmions. Sci. Rep. 5, 9400 (2015).

13. Song, K. M. et al. Skyrmion-based artificial synapses for neuromorphic computing. Nat. Electron. 3, 148-155 (2020).

14. Mühlbauer, S. et al. Skyrmion Lattice in a Chiral Magnet. Science 323, 915-919 (2009).

15. Yu, X. Z. et al. Real-space observation of a two-dimensional skyrmion crystal. Nature 465, 901-904 (2010).

16. Woo, S. et al. Observation of room-temperature magnetic skyrmions and their current-driven dynamics in ultrathin metallic ferromagnets. Nat. Mater. 15, 501-506 (2016).

17. Büttner, F. et al. Field-free deterministic ultrafast creation of magnetic skyrmions by spin-orbit torques. Nat. Nanotechnol. 12, 1040-1044 (2017).

18. Woo, S. et al. Deterministic creation and deletion of a single magnetic skyrmion observed by direct time-resolved X-ray microscopy. Nat. Electron. 1, 288-296 (2018).

19. Ritzmann, U. et al. Trochoidal motion and pair generation in skyrmion and antiskyrmion dynamics under spin-orbit torques. Nat. Electron. 1, 451-457 (2018).

20. Moreau-Luchaire, C. et al. Additive interfacial chiral interaction in multilayers for stabilization of small individual skyrmions at room temperature. Nat. Nanotechnol. 11, 444-448 (2016).

21. Zázvorka, J. et al. Thermal skyrmion diffusion used in a reshuffler device. Nat. Nanotechnol. 14, 658-661 (2019).

22. Hubert, A., Malozemoff, A. P. \& DeLuca, J. C. Effect of cubic, tilted uniaxial, and orthorhombic anisotropies on homogeneous nucleation in a garnet bubble film. J. Appl. Phys. 45, 3562-3571 (1974).

23. Choi, J. et al. Magnetic Bubble Domain Phase at the Spin Reorientation Transition of Ultrathin Fe/Ni/Cu(001) Film. Phys. Rev. Lett. 98, 207205 (2007).

24. $\mathrm{Yu}, \mathrm{X}$. et al. Aggregation and collapse dynamics of skyrmions in a nonequilibrium state. Nat. Phys. 14, 832-836 (2018).

25. Han, M.-G. et al. Scaling, rotation, and channeling behavior of helical and skyrmion spin textures in thin films of Te-doped Cu2OSeO3. Sci. Adv. 6, 13 (2020).

26. Dzyaloshinsky, I. A thermodynamic theory of "weak" ferromagnetism of antiferromagnetics. J. Phys. Chem. Solids 4, 241-255 (1958).

27. Je, S.-G. et al. Asymmetric magnetic domain-wall motion by the Dzyaloshinskii-Moriya interaction. Phys. Rev. B 88, 214401 (2013).

28. Ikeda, S. et al. A perpendicular-anisotropy CoFeB-MgO magnetic tunnel junction. Nat. Mater. 9, 721-724 (2010).

29. Yang, H. X. et al. First-principles investigation of the very large perpendicular magnetic anisotropy at Fe|MgO and ColMgO interfaces. Phys. Rev. B 84, 054401 (2011).

30. Saratz, N., Ramsperger, U., Vindigni, A. \& Pescia, D. Irreversibility, reversibility, and thermal equilibrium in domain patterns of Fe films with perpendicular magnetization. Phys. Rev. B 82, 184416 (2010).

31. Meier, T. N. G., Kronseder, M. \& Back, C. H. Domain-width model for perpendicularly magnetized systems with Dzyaloshinskii-Moriya interaction. Phys. Rev. B 96, 144408 (2017).

32. Kashuba, A. \& Pokrovsky, V. L. Stripe domain structures in a thin ferromagnetic film. Phys. Rev. Lett. 70, 3155-3158 (1993).

33. Meier, T. N. G., Kronseder, M., Zimmermann, M. \& Back, C. H. Quantification of thermal fluctuations in stripe domain patterns. Phys. Rev. B 93 064424 (2016).

34. Chaurasiya, A. K. et al. Direct Observation of Interfacial Dzyaloshinskii-Moriya Interaction from Asymmetric Spin-wave Propagation in W/CoFeB/SiO2 Heterostructures Down to Sub-nanometer CoFeB Thickness. Sci. Rep. 6, 32592 (2016).

35. Soucaille, R. et al. Probing the Dzyaloshinskii-Moriya interaction in CoFeB ultrathin films using domain wall creep and Brillouin light spectroscopy. Phys. Rev. B 94, 104431 (2016).

36. Yoshimura, Y. et al. Soliton-like magnetic domain wall motion induced by the interfacial Dzyaloshinskii-Moriya interaction. Nat. Phys. 12, 157-161 (2016). 
37. Büttner, F., Lemesh, I. \& Beach, G. S. D. Theory of isolated magnetic skyrmions: from fundamentals to room temperature applications. Sci. Rep. 8, 4464 (2018).

38. Woo, S. et al. Spin-orbit torque-driven skyrmion dynamics revealed by timeresolved X-ray microscopy. Nat. Commun. 8, 15573 (2017).

39. Montoya, S. A. et al. Spin-orbit torque induced dipole skyrmion motion at room temperature. Phys. Rev. B 98, 104432 (2018).
40. Ma, C. et al. Electric field-induced creation and directional motion of domain walls and skyrmion bubbles. Nano Lett. 19, 353-361 (2019).

41. Srivastava, T. et al. Large-voltage tuning of Dzyaloshinskii-Moriya interactions: a route toward dynamic control of skyrmoin chiralliy. Nano Lett. 18, 4871-4877 (2018). 